\title{
Arthritis in Kawasaki disease: A poorly recognised manifestation
}

\author{
Andreia Martins, ${ }^{1}$ Marta Conde ${ }^{2}$ Maria Brito ${ }^{1}$ and Catarina Gouveia ${ }^{1}$ \\ 1Paediatric Infectious Diseases Unit, and ²Paediatric Rheumatology Unit, Dona Estefania Hospital, Lisbon, Portugal
}

\begin{abstract}
Aim: To determine the prevalence of arthritis in Kawasaki disease (KD) and the clinical characteristics of children with KD and arthritis.
Methods: This was a single-centre, 15-year, retrospective study of children admitted with KD. Clinical features (including coronary involvement), laboratory results and treatment response were evaluated.

Results: Of 63 children with KD, 60.3\% were male, with a median of age of 2.0 years. Complete KD was found in $68.3 \%$. The time from symptom onset to treatment was 7.0 days (median); $30.7 \%$ had coronary artery aneurysms, from which $82.5 \%$ responded to intravenous immunoglobulin. During the course of their illness, eight children developed arthritis (12.7\%), which was early onset in six (75\%) and oligoarticular in five (62.5\%). The median number of joints was $3.5\left(P_{25}=1.3, P_{75}=17.0\right)$, and at least one large joint was affected. In all cases, the arthritis was self-limited and left no sequelae, lasting a median of 14 days and no longer than 22 days. KD children with arthritis were older $(P=0.025)$, and those with early-onset arthritis responded to first-line therapy, unlike the late-onset group $(P=0.018)$.
\end{abstract}

Conclusions: This study emphasises the value of a systematic articular examination of joints in KD. Refractory KD was observed in children with late-onset arthritis.

Key words: arthritis; intravenous immunoglobulin; Kawasaki disease.

\section{What is already known on this topic}

1 Arthritis has long been described in Kawasaki disease (KD) but has had a dramatic decrease since the intravenous immunoglobulin (IVIG) era.

2 Literature has shown that this subgroup of patients appears to have a more pronounced systemic inflammation and IVIG resistance (refractory KD).

\section{What this paper adds}

1 The prevalence of arthritis found in our cohort (12.7\%) was higher than reported by others (2-7.5\%) in the post-IVIG era.

2 The presence of early-onset arthritis is associated with a good response to first-line therapy.

3 Late-onset arthritis might be associated with refractory KD.
Kawasaki disease (KD) is an acute, self-limited, inflammatory, medium-vessel vasculitis of unknown aetiology that mainly affects young children. ${ }^{1,2}$ It is the most common cause of acquired heart disease in children in developed countries. ${ }^{1} \mathrm{KD}$ is characterised by fever, bilateral non-exudative conjunctivitis, erythema of the lips and oral mucosa, changes in the extremities, polymorphous rash and cervical lymphadenopathy. ${ }^{3}$ Coronary artery lesions appear in $15-25 \%$ of untreated children. ${ }^{1}$

KD may present with non-cardiac manifestations in almost every organ and system. ${ }^{1}$ Arthritis in KD has long been described and was reported in $31 \%$ of patients in the pre-intravenous immunoglobulin (IVIG) era. ${ }^{2}$ The prevalence decreased to $2-7.5 \%$ due to the anti-inflammatory effect of IVIG. ${ }^{2,4}$ Although joint involvement has not been specifically associated with a worst outcome, a body of literature has demonstrated that this subgroup of patients has a more

Correspondence: Dr Catarina Gouveia, Paediatric Infectious Diseases Unit, Hospital Dona Estefânia-Centro Hospitalar Lisboa Central, Rua Jacinta Marto, 1169-045 Lisbon, Portugal. Fax: +351 213126 630; email: cmfgouveia@gmail.com

Conflict of interest: None declared.

Accepted for publication 22 May 2018. pronounced systemic inflammation and IVIG resistance. ${ }^{2,5}$ Arthritis in $\mathrm{KD}$ is mainly described in case reports. ${ }^{2,6,7}$ It is not a major diagnostic criterion in KD. It is possible that the presence of arthritis in $\mathrm{KD}$ is missed as joints cannot be easily assessed in a febrile irritable child. ${ }^{4}$

The purpose of this study is to determine the prevalence of arthritis associated with $\mathrm{KD}$, its clinical pattern, treatment response and prognosis in relation to the coronary outcome.

\section{Methods}

\section{Subjects and data}

We did a retrospective single-centre study of children admitted to a Portuguese paediatric tertiary hospital with a diagnosis of $\mathrm{KD}$ during a 15-year period from January 1998 to December 2013. The diagnosis, treatment and management of KD were established according to the American Heart Association (AHA) Guidelines. ${ }^{1,8}$ Treatment included IVIG $(2 \mathrm{~g} / \mathrm{kg})$ and high-dose aspirin $(80-100 \mathrm{mg} / \mathrm{kg} /$ day) until the child is afebrile for $48-72 \mathrm{~h}$, reducing to low dose $(3-5 \mathrm{mg} / \mathrm{kg})$ until there is no evidence of coronary artery lesions by $6-8$ weeks. Non-responders to the first-line therapy received a second course of $2 \mathrm{~g} / \mathrm{kg}$ IVIG and, if still 
Table 1 Demographic and clinical features of the eight patients with Kawasaki disease (KD) and articular involvement

\begin{tabular}{|c|c|c|c|c|c|c|c|c|c|c|c|}
\hline Patient & Gender & $\begin{array}{l}\text { Age at } \\
\text { diagnosis, } \\
\text { years }\end{array}$ & $K D$ & $\begin{array}{c}\text { Illness to } \\
\text { diagnosis, } \\
\text { days }\end{array}$ & Treatment & $\begin{array}{c}\text { Illness to } \\
\text { arthritis, } \\
\text { days }\end{array}$ & $\begin{array}{c}\text { Joints, } \\
n\end{array}$ & $\begin{array}{c}\text { Onset } \\
\text { of arthritis }\end{array}$ & Large joints & Small joints & $\begin{array}{c}\text { Arthritis } \\
\text { duration, } \\
\text { days }\end{array}$ \\
\hline 1 & M & 10 & C & 14 & One dose IVIG & 2 & 1 & Early & One ankle & 0 & 8 \\
\hline 2 & $\mathrm{~F}$ & 2 & C & 6 & One dose IVIG & 5 & 2 & Early & One hip & $1 \mathrm{MCP}$ & 2 \\
\hline 3 & $\mathrm{~F}$ & 2 & C & 6 & One dose IVIG & 4 & 4 & Early & $\begin{array}{l}\text { Two shoulders, } \\
\text { two knees }\end{array}$ & 0 & ND \\
\hline 4 & $\mathrm{~F}$ & 3 & C & 7 & Two doses IVIG & 14 & 3 & Late & Two hips, one knee & 0 & 11 \\
\hline 5 & M & 3 & I & 14 & One dose IVIG & 3 & 1 & Early & One hip & 0 & 22 \\
\hline 6 & M & 4 & C & 6 & $\begin{array}{c}\text { Two doses IVIG, } \\
\text { one dose MP }\end{array}$ & 4 & 35 & $\begin{array}{l}\text { Early } \\
\text { and late }\end{array}$ & $\begin{array}{r}\text { Two wrists, one } \\
\text { hip, two knees }\end{array}$ & $\begin{array}{c}10 \mathrm{MCP} \\
10 \mathrm{PIP}, 10 \mathrm{DIP}\end{array}$ & 14 \\
\hline 7 & M & 5 & I & 17 & One dose IVIG & 8 & 18 & Early & $\begin{array}{l}\text { Two elbows, two } \\
\text { wrists, two knees, } \\
\text { two ankle }\end{array}$ & $10 \mathrm{MCP}$ & 14 \\
\hline 8 & M & 3 & C & 4 & $\begin{array}{c}\text { Two doses IVIG, } \\
\text { one dose MP }\end{array}$ & 11 & 14 & Late & Two elbows, two knees & $10 \mathrm{PIP}$ & 18 \\
\hline
\end{tabular}

C, complete; DIP, distal interphalangeal joint; F, female; I, incomplete; IVIG, intravenous immunoglobulin; M, male; MCP, metacarpophalangeal joint; MP, methylprednisolone; ND, not determined; PIP, proximal interphalangeal joint.

unresponsive, were subsequently treated with steroids. Cardiac involvement was defined according to AHA 1994 and 2004 guidelines. $^{1,8}$

A paediatric consultant in rheumatology examined all children with arthritis, defined as joint pain with limited range of motion and/or swelling and/or evidence of synovitis on ultrasonography. Oligoarticular involvement was considered if less than five active joints were present and polyarticular involvement for five or more active joints. ${ }^{9}$ Early- and late-onset arthritis were, respectively, established according to its onset, until or after the 10th day of illness. ${ }^{1}$

\section{Statistical analysis}

An exploratory analysis was carried out for all variables. Nonparametric $\chi^{2}$, Fisher's exact and Mann-Whitney tests were used, with a level of significance of $\alpha=0.05$. All data were analysed using SPSS, version 22.0 (IBM Corp., Armonk, NY, USA).

\section{Results}

Sixty-three children were diagnosed with KD; 38 (60.3\%) were male, and $41(83.7 \%)$ were Caucasian. The median age at diagnosis was 2.0 years (range 2.4 months-1 1.5 years). Complete KD was observed in 43 patients $(68.3 \%)$. The median time from symptom onset to definitive treatment was 7.0 days $\left(\mathrm{P}_{25}=5.0\right.$, $\left.P_{75}=9.0\right)$. Most patients responded to the first IVIG dose $(82.5 \%)$, 11 needed additional IVIG, 5 required steroids and 1 infliximab. The incidence of coronary artery aneurysms was 30.1\%.

Eight children developed arthritis during the course of their illness $(12.7 \%)$. Table 1 further describes the clinical features.

The median time lapse from symptom onset to the appearance of arthritis was 4.5 days $(\min =2.0 ; \max =14.0)$. Early-onset and late-onset arthritis were, respectively, observed in six $(75.0 \%)$ and three $(37.5 \%)$ patients (one patient had both presentations). The number of joints affected ranged from 1 to
35 , and at least one large joint was affected. Five $(62.5 \%)$ had oligoarticular involvement and three $(37.5 \%)$ polyarticular, in which small joints of the hand were always symmetrically involved.

The median duration of articular involvement was 14 days $\left(\mathrm{P}_{25}=8.0, \mathrm{P}_{75}=18.0\right)$, lasting no longer than 22 days. An additional non-steroidal anti-inflammatory drug (ibuprofen) was needed in patients with polyarticular presentation; however, only one (patient 8) required non-steroidal anti-inflammatory drugs after discharge. All patients with arthritis recovered, with no joint sequelae.

Regarding KD children with and without arthritis, those with articular involvement were older and had higher haemoglobin levels (Table 2). Three of the 8 children with arthritis (37.5\%) and 16 of the 55 children without arthritis had coronary artery involvement $(29.1 \%)$. The difference was not statistically significant.

Five of six patients with early-onset arthritis responded to the first IVIG course, unlike all patients with late-onset arthritis (patients 4, 6 and 8), who required a second IVIG course or even methylprednisolone $(P=0.018)$. Patient 4 had a complete $\mathrm{KD}$ and responded to a first IVIG course at day 8 . However, on day 14 , the fever relapsed, accompanied by oligoarthritis, and only remitted after a second IVIG course. Patient 6 had a mixed arthritic pattern, with acute left knee arthritis on day 4, which resolved a few days after a second IVIG dose. Nevertheless, fever persisted, and arthritis relapsed on day 11, involving 35 joints. Another patient (patient 8) did not respond after two IVIG courses and, on day 11 , presented with polyarthritis to a total joint count of 14. Both patients 6 and 8 needed a methylprednisolone course for complete recovery.

\section{Discussion}

$\mathrm{KD}$ is a multisystem vasculitis, affecting mostly medium-sized arteries. The articular involvement in $\mathrm{KD}$ has been reported, 
Table 2 Demographic and clinical characteristics of Kawasaki disease (KD) children, with and without arthritis

\begin{tabular}{|c|c|c|c|}
\hline & With arthritis, $n=8$ & No arthritis, $n=55$ & $P$ \\
\hline Age, years, median $\left(\mathrm{P}_{25} ; \mathrm{P}_{75}\right)$ & $3.0(2.0 ; 4.8)$ & $1.5(0.8 ; 3.0)$ & $0.026+\hbar$ \\
\hline Male gender, $n(\%)$ & $5(62.5)$ & $33(60.0)$ & $1.000 \S$ \\
\hline Days of illness to treatment, median $\left(P_{25} ; P_{75}\right)$ & $6.5(6.0 ; 14.0)$ & $7.0(6.0 ; 9.0)$ & $0.510 \dagger$ \\
\hline Complete KD, $n(\%)$ & $6(75.0)$ & $37(67.3)$ & $1.000 \ddagger$ \\
\hline Coronary involvement, $n(\%)$ & $3(37.5)$ & $16(29.1)$ & $0.688+$ \\
\hline CRP, mg/L, median $\left(\mathrm{P}_{25} ; \mathrm{P}_{75}\right)$ & $111(82.5 ; 161,8)$ & $116(73.0 ; 150.0)$ & $0.496 \dagger$ \\
\hline ESR, mm/h, median $\left(P_{25} ; P_{75}\right)$ & $58.8(36.3 ; 104.3)$ & $101(70.0 ; 111.0)$ & $0.079 \dagger$ \\
\hline Haemoglobin, g/L, median $\left(\mathrm{P}_{25} ; \mathrm{P}_{75}\right)$ & $11.4(11.1 ; 11.8)$ & $10.6(10.2 ; 11.3)$ & $0.021+\hbar$ \\
\hline WBC, $\mu \mathrm{L}^{-1}$, median $\left(\mathrm{P}_{25} ; \mathrm{P}_{75}\right)$ & 14070 (12 422; 16 020) & 15350 (12 680; 20 000) & $0.302 \dagger$ \\
\hline Platelets, $\mu \mathrm{L}^{-1}$, median $\left(\mathrm{P}_{25} ; \mathrm{P}_{75}\right)$ & 353000 (134 000; 442 750) & 454000 (260 200; 524 000) & $0.077 \dagger$ \\
\hline Need for second IVIG, $n(\%)$ & $3(37.5)$ & $8(14.5)$ & $0.137 \ddagger$ \\
\hline Need for MP, $n(\%)$ & $2(25.0)$ & $2(3.6)$ & 0.075 \\
\hline
\end{tabular}

$\dagger$ Exact Mann-Whitney test $P$ value. $\ddagger$ Statistically significant values. §Fisher’s exact test $P$ value. CRP, C-reactive protein at admission; ESR, erythrocyte sedimentation rate at admission; IVIG, intravenous immunoglobulin; MP, methylprednisolone; WBC, white blood cells.

although poorly characterised. ${ }^{1}$ The prevalence of arthritis in KD arthritis has decreased dramatically since the introduction of IVIG therapy, making it even more difficult to describe. ${ }^{2,4}$ Interestingly, the prevalence of arthritis found in our cohort $(12.7 \%)$, although higher than reported by others $(2-7.5 \%)$, was significantly lower in comparison to the prevalence in pre-IVIG era $(31 \%)^{2}$

Arthritis in KD is generally self-limited, and intensely painful, conditioning a limping gait. ${ }^{2}$ Affected children are reported to have the same demographic features of children without arthritis; however, our data showed that arthritis in KD mainly affected older children.

In the pre-IVIG era, early-onset polyarthritis was more common; it developed during the first 10 days of illness and tended to involve multiple joints, including small and large weightbearing joints. ${ }^{9}$ This type of arthritis is often undistinguishable from systemic juvenile idiopathic arthritis. ${ }^{7,10}$ Late-onset arthritis (sub-acute stage) occurs after the 10th day of illness and is mainly oligoarticular, involving large weight-bearing joints. ${ }^{1,2}$ In our study, early-onset arthritis was the most frequent presentation; an oligoarticular pattern was dominant. In a remarkable fashion, one patient presented with a biphasic pattern, earlyonset arthritis was followed by late-onset arthritis, as already been reported by Duzova et al. ${ }^{11}$

It has been suggested that arthritis in $\mathrm{KD}$ might lead to a worse outcome $e^{7,10}$ because it indicates a more intense inflammatory response, ${ }^{2}$ accounting for some of the first-line therapy resistance. ${ }^{5}$ However, Gong et al. did not find any association between the articular involvement and worse prognosis or higher inflammatory response. ${ }^{2}$ Curiously, we found higher haemoglobin levels at admission in patients with arthritis, a finding also noted by Gong et al. ${ }^{2}$ We acknowledge that this may be due to the fact that haemoglobin level is influenced by age, with higher levels in older children.

Early-onset arthritis in KD has been reported to correlate with a worse outcome. ${ }^{11}$ However, in our cohort, five of six patients with early-onset arthritis responded to the first course of IVIG. Instead, late-onset arthritis was associated with refractory KD. Our study suggests that first-line therapy with IVIG is indicated irrespective of articular involvement in $\mathrm{KD}$ and that the presence of late-onset arthritis in refractory KD should be taken into consideration when choosing the second line of treatment.

Further studies are required to ascertain the role of arthritis in refractory $\mathrm{KD}$ in the determination of the most appropriate second line of treatment. In our study, arthritis in $\mathrm{KD}$ was not associated with the increased risk of coronary artery involvement.

Our study has several limitations. It is a retrospective analysis of a small-sized sample, and the diagnostic criteria for coronary artery abnormalities according to AHA guidelines changed over the study period. Finally, we cannot rule out if arthritis was underestimated in younger children as it is difficult to diagnose arthritis in this age group, especially in the presence of swelling and induration of hands and feet.

\section{Conclusions}

This study shows the importance of a systematic evaluation of articular involvement in KD. We suggest that, in general, earlyonset arthritis is associated with a good response to the first-line therapy in $\mathrm{KD}$ and that late-onset arthritis might indicate refractory KD.

\section{References}

1 Newburger JW, Takahashi M, Gerber MA et al. Diagnosis, treatment, and long-term management of Kawasaki disease: A statement for health professionals from the Committee on Rheumatic Fever, Endocarditis, and Kawasaki Disease, Council on Cardiovascular Disease in the Young, American Heart Association. Pediatrics 2004; 114: 1708-33.

2 Gong GWK, McCrindle BW, Ching JC, Yeung RSM. Arthritis presenting during the acute phase of Kawasaki disease. J. Pediatr. 2006; 148: 800-5.

3 McCrindle BW, Rowley AH, Newburger JW et al. Diagnosis, treatment, and long-term management of Kawasaki disease: A scientific statement for health professionals from the American Heart Association. Circulation 2017; 135: e927-99.

4 Lee KY, Oh JH, Han JW, Lee JS, Lee BC. Arthritis in Kawasaki disease after responding to intravenous immunoglobulin treatment. Eur. J. Pediatr. 2005; 164: 451-2. 
5 Hartas GA, Hashmi SS, Pham-Peyton C, Tsounias E, Bricker JT, GuptaMalhotra M. Immunoglobulin resistance in Kawasaki disease. Pediatr. Allergy Immunol. Pulmonol. 2015; 28: 13-9.

6 Bowler J, Drvaric DM, Roberts JM, Burke SW. Kawasaki syndrome presenting as pyarthrosis of the hip. A case report. J. Bone Joint Surg. Am. 1986; 68: 467-8.

7 Dong S, Bout-Tabaku S, Texter K, Jaggi P. Diagnosis of systemic-onset juvenile idiopathic arthritis after treatment for presumed Kawasaki disease. J. Pediatr. 2015; 166: 1283-8.

8 Dajani AS, Taubert KA, Takahashi M et al. Guidelines for long-term management of patients with Kawasaki disease. Report from the Committee on Rheumatic Fever, Endocarditis, and Kawasaki Disease,
Council on Cardiovascular Disease in the Young, American Heart Association. Circulation 1994; 89: 916-22.

9 Petty RE, Southwood TR, Manners P et al. International League of Associations for Rheumatology classification of juvenile idiopathic arthritis: Second revision, Edmonton, 2001. J. Rheumatol. 2004; 31: $390-2$.

10 Rigante $\mathrm{D}$, Valentini $\mathrm{P}$, Onesimo $\mathrm{R}$ et al. Incomplete Kawasaki syndrome followed by systemic onset-juvenile idiopathic arthritis mimicking Kawasaki syndrome. Rheumatol. Int. 2010; 30: 535-9.

11 Duzova A, Topaloglu R, Keskin M, Ozcelik U, Secmeer G, Tokgozoglu AM. An unusual pattern of arthritis in a child with Kawasaki syndrome. Clin. Rheumatol. 2004; 23: 73-5.
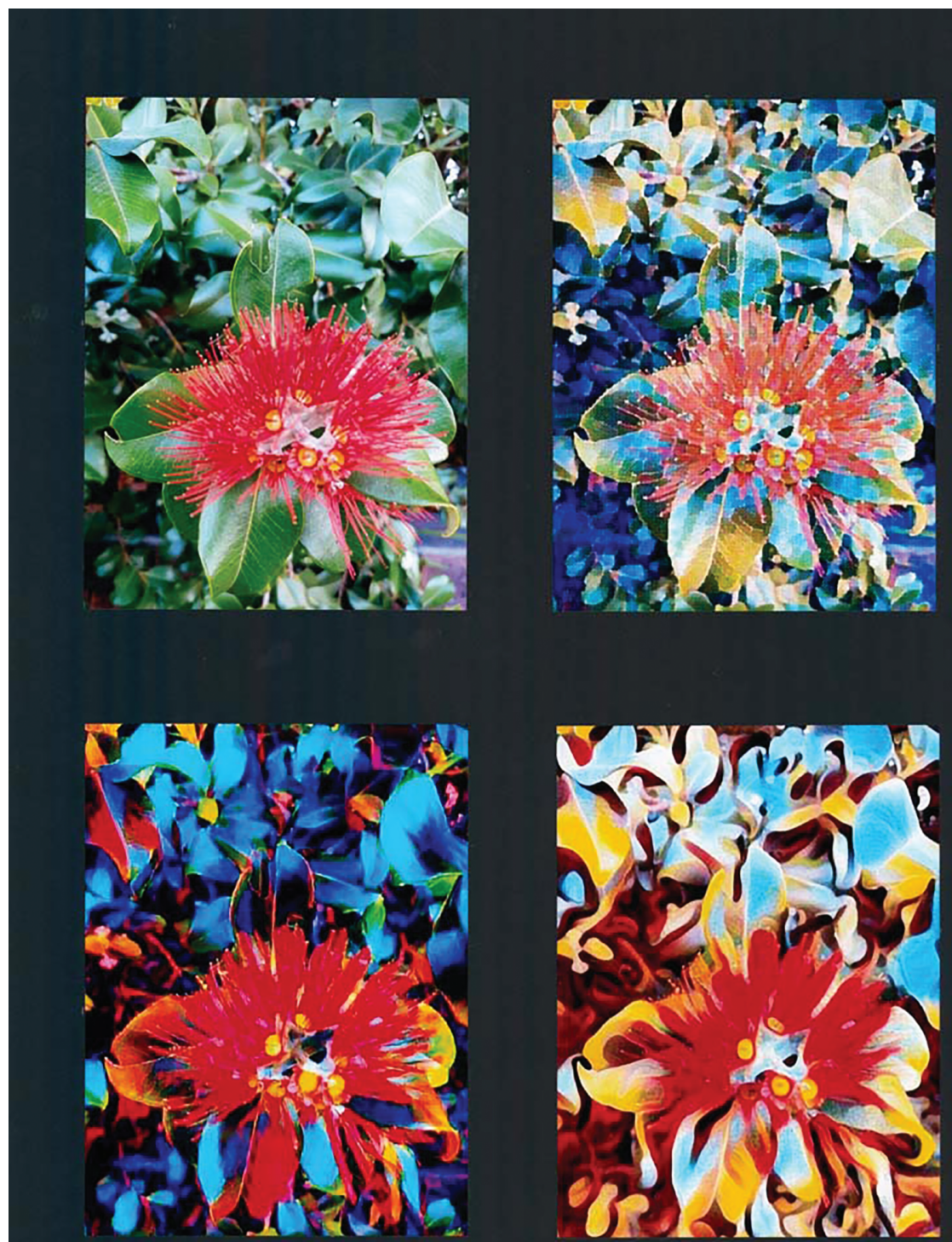

Four ways by Mohammad Zain, Saeed Khayal and Nathaniel Ledua (all age 5) from Operation Art 2017 